\title{
A Discretized Tikhonov Regularization Method for a Fractional Backward Heat Conduction Problem
}

\author{
Zhi-Liang Deng ${ }^{1}$ and Xiao-Mei Yang ${ }^{2}$ \\ ${ }^{1}$ School of Mathematical Sciences, University of Electronic Science and Technology of China, Chengdu 610054, China
}

${ }^{2}$ School of Mathematics, Southwest Jiaotong University, Chengdu 610031, China

Correspondence should be addressed to Zhi-Liang Deng; dengzhl@uestc.edu.cn

Received 30 December 2013; Accepted 7 February 2014; Published 18 March 2014

Academic Editor: Ming Li

Copyright (c) 2014 Z.-L. Deng and X.-M. Yang. This is an open access article distributed under the Creative Commons Attribution License, which permits unrestricted use, distribution, and reproduction in any medium, provided the original work is properly cited.

We propose a numerical reconstruction method for solving a time-fractional backward heat conduction problem. Based on the idea of reproducing kernel approximation, we reconstruct the unknown initial heat distribution from a finite set of scattered measurements of transient temperature at a fixed final time. The standard Tikhonov regularization technique using the norm of reproducing the kernel Hilbert space as the penalty term is adopted to provide a stable solution when the measurement data contains noise. Numerical results indicate that the proposed method is efficient.

\section{Introduction}

Let $\Omega \subset \mathbb{R}^{d}, d \in \mathbb{N}$, be a bounded domain with sufficiently smooth boundary $\partial \Omega$. Consider the following initial boundary value problem for time-fractional diffusion equation (TFDE):

$$
\begin{gathered}
{ }_{0} D_{t}^{\gamma} u(x, t)=\mathscr{L} u, \quad x \in \Omega, t \in[0, T], \\
u(x, 0)=f(x), \quad x \in \bar{\Omega}, \\
\mathscr{B} u(x, t):=\mu u(x, t)+\beta \frac{\partial u}{\partial v}=0, \quad x \in \partial \Omega, t \in[0, T] .
\end{gathered}
$$

Here ${ }_{0} D_{t}^{\gamma}$ denotes the Caputo fractional derivative with respect to $t$ and is defined by

$$
{ }_{0} D_{t}^{\gamma} \psi(t)= \begin{cases}\frac{1}{\Gamma(1-\gamma)} \int_{0}^{t} \frac{\psi^{\prime}(\tau)}{(t-\tau)^{\gamma}} d \tau, & 0<\gamma<1, \\ \psi^{\prime}(t), & \gamma=1 .\end{cases}
$$

$\Gamma(\cdot)$ is the Gamma function and $\mathscr{L}$ is a symmetric uniformly elliptic operator and $T>0$ is a fixed final time, $\mu$ and $\beta$ are constants, and $v$ is the outward unit normal vector of the domain $\Omega$. In what follows, let $0<\gamma<1$ and $\mathscr{L}$ be given by

$$
\begin{aligned}
\mathscr{L} u(x):=\sum_{i=1}^{d} \frac{\partial}{\partial x_{i}}\left(\sum_{j=1}^{d} D_{i j}(x) \frac{\partial}{\partial x_{j}} u(x)\right) \\
+Q(x) u(x), \quad x \in \Omega,
\end{aligned}
$$

where $Q \in C(\bar{\Omega}), Q(x) \leq 0$ for $x \in \bar{\Omega}$ and $D_{i j}=D_{j i} \in C^{1}(\bar{\Omega})$, $1 \leq i, j \leq d$. Moreover, we assume that there exists a positive constant $D>0$ such that

$$
D \sum_{i=1}^{d} \eta_{i}^{2} \leq \sum_{i, j=1}^{d} D_{i j}(x) \eta_{i} \eta_{j}, \quad x \in \bar{\Omega}, \eta \in \mathbb{R}^{d} .
$$

Recently, people are shifting their partial focus to fractional-order differential equations (FDEs) with the realization that the use of fractional-order derivatives and integrals leads to formulas of certain physical processes (for instance, some anomalous diffusion processes) which is more economical and useful than the classical approach in terms of Fick's laws of diffusion. In this paper, we consider the fractional-order partial differential equation (FPDE) in (1), which is obtained from the standard diffusion equation by replacing the first-order time derivative with a fractional 
derivative of order $\gamma$, with $0<\gamma<1$. Different models using this kind of FDEs have been proposed [1-3], and there has been significant interest in developing numerical schemes for their solution. Physically, the time-fractional partial differential equations describe the continuous time random walk problems (the non-Markovian process). The physical interpretation of the fractional derivative is that it represents a degree of memory in the diffusing material [4]. Actually, the convolution integral in the definition of the Caputo fractional-order derivatives for $\gamma \in(0,1)$ at time $t$ requires all the knowledge of classical derivative $\psi^{\prime}(\tau)$ for $\tau \in(0, t)$, which reflects the "memory effect" of fractional derivatives. The utilization of the memory effect of fractional derivatives comes with a high cost regarding numerical solvability. Any algorithm using a discretization of a noninteger derivative has to take into account its nonlocal structure which means in general a high storage requirement and great overall complexity of the algorithm. Similar convolution model can be used to depict the tumor-immune system [5]. Fractional dimensional model is also used to characterize the binary images of DNA [6] and oscillators [7, 8]. In [9], a general approach is proposed to approximate ideal filters based on fractional calculus from the point of view of systems of fractional order. Recently, numerous attempts to solve TFDE can be found in the literature $[4,10-14]$. We can refer to [1518] for more specified knowledge about fractional calculus.

The backward problem governed by time-fractional partial differential equation in (1) is to recover the heat distribution at any earlier time $0 \leq t<T$ from the measurement $u_{\epsilon}(x, T)$, written as $u_{T}^{\epsilon}$, which is noise-contaminated data for the exact temperature $u(x, T)$ :

$$
\left\|u_{\epsilon}(\cdot, T)-u(\cdot, T)\right\|_{L^{2}(\Omega)} \leq \epsilon
$$

for some known noise level $\epsilon>0$. Such inverse problems have been considered by several authors. Based on the eigenfunction expansions, Sakamoto and Yamamoto [19] establish the unique existence of the weak solution and the asymptotic behavior as the time $t$ goes to $\infty$ for the forward problem and prove the stability and uniqueness in the backward problem in time. For the one-dimensional case, Liu and Yamamoto [20] propose a regularizing scheme by the quasi-reversibility to restore the stability of the backward problem. In [21], a regularization by projection is applied to the same problem as in [20] and the corresponding convergence rates are obtained under a priori and a posteriori parameter choice strategies, respectively. Here, we pay our attention to the situation of stable reconstruction of the initial heat distribution $f(x)$ from some scattered noisy data of $u_{\epsilon}(\cdot, T)$. More specifically, the data $u_{\epsilon}(\cdot, T)$ are collected only at a finite set of points $\left\{z_{1}, z_{2}, \ldots, z_{m}\right\} \in \Omega$. We then reconstruct the initial temperature distribution $u(x, 0)$ from the scattered noisy data at $t=T$. For solving the backward diffusion problem, we employ a discretized Tikhonov regularization by the Ritz approach coupled with the reproducing kernel Hilbert space (RKHS), which is proposed in [22].

The Tikhonov regularization method has been widely studied and applied to all varieties of ill-posed problems $[23,24]$. The discretized Tikhonov regularization method and its relative theories are also explored in detail [24]. We adopt the Tikhonov regularization method by a reproducing kernel Hilbert space into the backward problem (1). As we know, the theory and practice of reproducing kernel are a fast growing research area. The numerical methods by RKHS have been also rapidly developed in recent years $[25,26]$. These developments are due to the increasing interest in the use of reproducing kernel for the solution of mathematical and engineering problems, for instance, machine learning [27], signal processing [28], stochastic processes [29], wavelet transforms [30], and so forth. For the details of RKHS, we are able to refer to [31]. However, to the authors' knowledge, there are few applications of RKHS to inverse problems. We provide the partial list of the recent works. Takeuchi and Yamamoto [22] prove the convergence of the discretized Tikhonov regularization method by RKHS. Hon and Takeuchi [32] apply this method into a backward heat conduction problem for parabolic-type partial differential equations. In reproducing the kernel Hilbert space settings, an inverse source identification problem for parabolic equations is considered in [33]. In [34], Saitoh discusses comprehensively the corresponding applications of RKHS in inverse problems.

The remainder of this paper is composed of five sections. In Section 2, we discuss Green's function for problem (1) and use it to construct the reproducing kernel. In Section 3, we state the reconstruction method of the fractional backward diffusion problem by using the reproducing kernel. In Section 4, some numerical examples are given to illustrate the effectiveness of our method. A summary is made in the Section 5. Finally, we list some existing knowledge about the reproducing kernel Hilbert space in the Appendix.

\section{Green's Function and the Reproducing Kernel}

In this section, we explore Green's function of system (1) and use it to construct the reproducing kernel. Firstly, let function $G(x, t ; \xi)$ be Green's function of the system (1); that is, $G$ satisfies the following equations in distribution's sense:

$$
\begin{gathered}
{ }_{0} D_{t}^{\gamma} G(x, t ; \xi)=\mathscr{L} G(x, t ; \xi), \quad x, \xi \in \Omega, t \in[0, T], \\
G(x, 0 ; \xi)=\delta(x-\xi), \quad x, \xi \in \Omega, \\
\mathscr{B} G(x, t ; \xi):=\mu G(x, t ; \xi)+\beta \frac{\partial G}{\partial \nu}=0, \quad x \in \partial \Omega, t \in[0, T] .
\end{gathered}
$$

It is easy to know that problem (6) is equivalent to the following problem:

$$
\begin{gathered}
{ }_{0} D_{t}^{\gamma} G(x, t ; \xi)-\mathscr{L} G(x, t ; \xi)=\delta(x-\xi) \delta(t), \\
x, \xi \in \Omega, t \in[0, T], \\
G(x, 0 ; \xi)=0, \quad x, \xi \in \Omega, \\
\mathscr{B} G(x, t ; \xi) \quad x \in \partial \Omega, t \in[0, T] .
\end{gathered}
$$


Let us employ the Laplace transform to solve the system (6). The Laplace transform of a function $\varphi$ on $\mathbb{R}^{+}$is defined by

$$
\phi(s):=\int_{0}^{\infty} e^{-s t} \varphi(t) d t, \quad \operatorname{Re}(s)>0 .
$$

The Laplace transform of the Caputo fractional derivative is given by [10]

$$
\int_{0}^{\infty} e_{0}^{-s t} D_{t}^{\gamma} \varphi(t) d t=s^{\gamma} \phi(s)-\sum_{k=0}^{J-1} s^{\gamma-k-1}{ }_{0} D_{t}^{k} \varphi(0)
$$

where $J-1<\gamma \leq J$. The Caputo fractional derivative appears more suitable to be treated by the Laplace transforming in that it requires the knowledge of initial values of the function and of its integer derivatives of order $k=1,2, \ldots, J-1$. By the Laplace transforming about the time variable $t$, the system (6) becomes

$$
\begin{gathered}
s^{\gamma} g(x, s ; \xi)-s^{\gamma-1} \delta(x-\xi) \\
=\mathscr{L} g(x, s ; \xi), \quad x, \xi \in \Omega, \operatorname{Re}(s)>0, \\
\mu g(x, s ; \xi)+\beta \frac{\partial g}{\partial \nu}(x, s ; \xi)=0,
\end{gathered}
$$

where $g$ denotes the Laplace transform of Green's function $G$. It should be noted here that the system in (1) is only defined on $t \in[0, T]$, not on $[0,+\infty)$. When we make the Laplace transform, some necessary preprocess, for example, the function continuation technique, needs to be done on the solution of (1) to satisfy the condition of the Laplace transform. Because we do not use the value of $u$ for $t>T$, the condition can be satisfied easily.

Applying this technique of eigenfunction expansions to problems (10), we have that

$$
g(x, s ; \xi)=\sum_{n=1}^{\infty} \frac{s^{\gamma-1}}{s^{\gamma}+\lambda_{n}^{2}} \varphi_{n}(\xi) \varphi_{n}(x),
$$

where $\varphi_{n}(x)$ is the $n$th orthonormal eigenfunction and $\lambda_{n}$ is the corresponding eigenvalue to the Sturm-Liouville problem

$$
\mathscr{L} \varphi(x)+k^{2} \varphi(x)=0
$$

subject to the boundary conditions

$$
\mu \varphi(x)+\beta \frac{\partial \varphi}{\partial \nu}=0 .
$$

Taking the Laplace inverse of (11), we have that

$$
G(x, t ; \xi)=\sum_{n=1}^{\infty} E_{\gamma}\left(-\lambda_{n}^{2} t^{\gamma}\right) \varphi_{n}(\xi) \varphi_{n}(x),
$$

where $E_{\gamma}(z)$ is the Mittag-Leffler function defined by

$$
E_{\gamma}(z)=\sum_{k=0}^{\infty} \frac{z^{k}}{\Gamma(\gamma k+1)}
$$

For the details of the Mittag-Leffler function, one can refer to [3]. Subsequently, one can easily verify that the unique solution of system (1) with initial value $u(x, 0)=f \in H$ can be written as

$$
u(x, t)=\int_{\Omega} G(x, t ; \xi) f(\xi) d \xi
$$

where $G(x, t ; \xi)$ is Green's function defined by (14). With the aid of (16), for each $f \in L^{2}(\Omega)$, introducing the operator $K$ : $L^{2}(\Omega) \rightarrow L^{2}(\Omega)$ by

$$
K f(x):=\int_{\Omega} G(x, T ; \xi) f(\xi) d \xi,
$$

we may formulate the inverse problem as an integral equation of the first kind

$$
K f(x)=u(x, T) .
$$

The symmetry of Green's function indicates that the operator $K$ is self-adjoint. We proceed by giving a brief account on the close connections of the ill posedness of operator equation (18) with the singular value system of operator $K$. According to the symmetry of operator $K$, we only need to discuss its eigensystem. It is easy to know that the eigensystem of $K$ is given by

$$
\left\{E_{\gamma}\left(-T^{\gamma} \lambda_{n}^{2}\right), \varphi_{n}\right\}_{n=1}^{\infty}
$$

where $E_{\gamma}\left(-T^{\gamma} \lambda_{n}^{2}\right)$ is the eigenvalue and $\varphi_{n}$ is the corresponding eigenfunction. We can see the decay of the eigenvalues $\left\{E_{\gamma}\left(-T^{\gamma} \lambda_{n}^{2}\right)\right\}_{n=1}^{\infty}$ with the increase of $\lambda_{n}$ from the following asymptotic behavior of the Mittag-Leffler function $E_{\gamma}(\cdot)$ which can be found in $[20,21]$ or by the results in [3].

Lemma 1 (see [20]). Let $0<\gamma_{0}<\gamma_{1}<1$. Then there exist constants $C_{1, \pm}, C_{2, \pm}>0$ depending only on $\gamma_{0}$ and $\gamma_{1}$ such that

$$
\begin{gathered}
\frac{C_{1,-}}{\gamma} e^{x^{1 / \gamma}} \leq E_{\gamma}(x) \leq \frac{C_{1,+}}{\gamma} e^{x^{1 / \gamma}}, \quad \forall x \geq 0, \\
\frac{C_{2,-}}{\Gamma(1-\gamma)} \frac{1}{1-x} \leq E_{\gamma}(x) \leq \frac{C_{2,+}}{\Gamma(1-\gamma)} \frac{1}{1-x}, \quad \forall x \leq 0 .
\end{gathered}
$$

These estimates are uniform for all $\gamma \in\left[\gamma_{0}, \gamma_{1}\right]$.

Next, we consider the construction of reproducing kernel using Green's function $G(x, t ; \xi)$. Define $\Phi(x, \xi):=G\left(x, t_{0} ; \xi\right)$ for some $t_{0} \in(0, T)$. The symmetry about the space variable $x, \xi$ of Green's function indicates that $\Phi(\cdot, \cdot): \Omega \times \Omega \rightarrow \mathbb{R}$ is symmetric. Now that $\Phi$ is a symmetric positive definite kernel, a unique RKHS in which the given kernel acts as the reproducing kernel can be constructed (see [26] for details). Henceforth we denote by $H_{t_{0}}$ the RHKS generated by the kernel $\Phi(x, \xi)=G\left(x, t_{0} ; \xi\right)$. Actually, according to [26, Chapter 10], the inner product and norm on $H_{t_{0}}$ are defined by

$$
\begin{gathered}
(f, g)_{H_{t_{0}}}:=\sum_{n=1}^{\infty} \frac{1}{E_{\gamma}\left(-\lambda_{n}^{2} t_{0}^{\gamma}\right)}\left(f, \varphi_{n}\right)_{L^{2}(\Omega)}\left(g, \varphi_{n}\right)_{L^{2}(\Omega)}, \\
\|f\|_{H_{t_{0}}}^{2}:=\sum_{n=1}^{\infty} \frac{1}{E_{\gamma}\left(-\lambda_{n}^{2} t_{0}^{\gamma}\right)}\left|\left(f, \varphi_{n}\right)_{L^{2}(\Omega)}\right|^{2},
\end{gathered}
$$


respectively. The space $H_{t_{0}}$ is actually given by

$$
H_{t_{0}}=\left\{f \in L^{2}(\Omega): \sum_{n=1}^{\infty} \frac{1}{E_{\gamma}\left(-\lambda_{n}^{2} t_{0}^{\gamma}\right)}\left|\left(f, \varphi_{n}\right)_{L^{2}(\Omega)}\right|^{2}<\infty\right\} .
$$

The second inequalities in Lemma 1 show that, as the function of $\lambda_{n}, 1 / E_{\gamma}\left(-\lambda_{n}^{2} t_{0}^{\gamma}\right) \in \mathcal{S} \mathscr{I}$, where $\mathcal{S} \mathscr{I}$ denotes the collection of slowly increasing functions [26] defined by

$$
\begin{aligned}
\mathcal{S} \mathscr{I}:=\left\{f: \mathbb{R}^{d}\right. & \longrightarrow \mathbb{R} \mid f(x)=O\left(\|x\|_{2}^{m}\right) \\
\text { as }\|x\|_{2} & \left.\longrightarrow \infty \text { for some } m \in \mathbb{N}_{0}\right\} .
\end{aligned}
$$

Hence, according to the theoretical results of [26, Chapter 10], we assert that the space $H_{t_{0}}$ is consistent with some Sobolev space $H^{k}(\Omega)$ for $k>d / 2$ and the norm on $H_{t_{0}}$ is equivalent to the norm $\|f\|_{H^{k}(\Omega)}=\left[\sum_{|\alpha| \leq k}\left\|D^{\alpha} f\right\|_{L^{2}(\Omega)}^{2}\right]^{1 / 2}$.

\section{Formulation of the Inverse Problem and the Reconstruction Method}

In order to find the initial temperature distribution $f(x)$, we would like to determine the solution of minimization problem

$$
\inf _{f \in L^{2}(\Omega)}\left\|K f-u_{\epsilon}(\cdot, T)\right\|_{L^{2}(\Omega)} .
$$

However, the minimization element of (25) generally is a poor approximation of the desired initial function $f$ due to the error $\epsilon$ in $u(x, T)$ and the ill posedness of operator equation (18). The Tikhonov regularization replaces the minimization problem (25) by the solution of a penalized least-squares problem

$$
\inf _{f \in H_{t_{0}}(\Omega)} J_{\alpha}(f):=\inf _{f \in H_{t_{0}}(\Omega)}\left\|K f-u_{\epsilon}(\cdot, T)\right\|_{L^{2}(\Omega)}^{2}+\alpha\|f\|_{H_{t_{0}}}^{2}
$$

with regularization parameter $\alpha>0$. We can see from [23, Proposition 3.11] that the convergence of any regularization method can be arbitrarily slow in general. Actually, convergence rates can be given only on some subset of $H_{t_{0}}$, that is, under a priori assumptions on the exact data. Here, we assume the exact solution $f$ belongs to the set of source conditions

$$
\mathscr{M}_{\mu}:=\left\{f \in H_{t_{0}}(\Omega): f=\left(K^{*} K\right)^{\mu} \omega,\|\omega\|_{L^{2}(\Omega)} \leq E\right\},
$$

where $E$ is the a priori bound and $\mu>0$ is a constant. As in [23], we know that there exists a constant $\mu_{0}$, named the "qualification" of the regularization method, such that $0<\mu \leq \mu_{0}$. For the Tikhonov regularization method, the qualification $\mu_{0}=1$. However, according to (21), it is easy to know that $\mathscr{M}_{\mu_{2}} \subset \mathscr{M}_{\mu_{1}}$ as $\mu_{1}>\mu_{2}$ and $\mathscr{M}_{1 / 4}=H_{t_{0}}$. Therefore, here we only need consider the case of $0<\mu \leq 1 / 4$ for the Tikhonov method (26).
In order to solve the minimization problem (26), some discretization scheme needs to be given. A natural way to obtain such discretization is to generate a finite dimensional approximation to the minimal element of the Tikhonov functional $J_{\alpha}$. For this, we define a subspace $V_{N}:=\operatorname{span}\{\Phi(\cdot, \xi)$ : $\left.\xi \in X_{N}\right\} \subset H_{t_{0}}$, where $X_{N}:=\left\{\xi_{1}, \xi_{2}, \ldots, \xi_{N}\right\} \subset \Omega$. This approximation by discretization is equivalent to finding the minimal norm least-squares solution of the equation

$$
K_{N} f:=K P_{N} f=u_{T},
$$

where $P_{N}: H_{t_{0}} \rightarrow V_{N}$ is the projection operator. Moreover, we produce a finite dimensional approximation $f_{\alpha, N}$ to $K^{\dagger} u_{T}$ by minimizing the Tikhonov functional (26) over the finite dimensional space $V_{N}$. Denote by $f_{\alpha, N, \epsilon}$ the minimizer of $J_{\alpha}$ for noise input data $u_{T}^{\epsilon}$. It is well known that $f_{\alpha, N, \epsilon}$ satisfy [23, 24]

$$
f_{\alpha, N, \epsilon}=\left(K_{N}^{*} K_{N}+\alpha I\right)^{-1} K_{N}^{*} u_{T}^{\epsilon}
$$

Provided $\left\{V_{N}\right\}$ is an expanding sequence, the convergence of the Tikhonov regularized solutions is proved [24]. Takeuchi and Yamamoto [22] show that the discretized Tikhonov regularized solutions converge to the exact solution without the monotonicity of $V_{N}$ under an a priori choice strategy for $N$ and $\alpha$. However, one can see that, from the existing results, in both cases, the regularized solutions $f_{\alpha, N}$ converging to the exact solution depends on whether $r_{N}:=\left\|K\left(I-P_{N}\right)\right\| \rightarrow$ $0,(N \rightarrow \infty)$. Moreover, the convergence of $r_{N}$ requires the knowledge of the fill distance $h_{X_{N}, \Omega}$, which is defined by [26]

$$
h_{X_{N}, \Omega}:=\sup _{x \in \Omega} \min _{\xi_{k} \in X_{N}}\left\|x-\xi_{k}\right\|
$$

The fill distance can be interpreted in various geometrical ways. For example, we can consider it as the radius of the largest ball which is completely contained in $\Omega$ and which does not contain a data site. In this sense $h_{X_{N}, \Omega}$ describes the largest data-site-free hole in $\Omega$.

Here, we utilize the same proof as in [32] to give the following lemma.

Lemma 2. Consider $r_{N}=\left\|K\left(I-P_{N}\right)\right\| \rightarrow 0$ as $N \rightarrow \infty$.

Proof. Consider

$$
\left\|K\left(I-P_{N}\right)\right\|=\sup _{\substack{f \in H_{t_{0}} \\ f \neq \theta}} \frac{\left\|K\left(I-P_{N}\right) f\right\|_{L^{2}(\Omega)}}{\|f\|_{H_{t_{0}}}} .
$$

It is obvious that the kernel function $\Phi(x, y)$ is sufficiently smooth. Therefore, according to the error estimate in the Appendix, we can find a positive constant $k$ such that the estimate

$$
\left\|f-P_{N} f\right\|_{L^{\infty}(\Omega)} \leq h_{X_{N}, \Omega}^{k}\|f\|_{H_{t_{0}}}
$$


holds for all $f \in H_{t_{0}}$. Meanwhile, it is easy to know

$$
\begin{aligned}
\|K f\|_{L^{2}(\Omega)} & =\left[\int_{\Omega}|K f|^{2} d x\right]^{1 / 2} \\
& =\left[\int_{\Omega}\left|\int_{\Omega} G(x, T ; \xi) f(\xi) d \xi\right|^{2} d x\right]^{1 / 2} \\
& \leq C\|f\|_{L^{\infty}(\Omega)}, \quad \forall f \in H_{t_{0}},
\end{aligned}
$$

where $C:=\left\{\int_{\Omega}\left[\int_{\Omega}|G(x, T ; \xi)| d \xi\right]^{2} d x\right\}^{1 / 2}$ is a constant. Moreover, the property of RKHS, $f(x)=(f(\cdot), \Phi(x, \cdot))$, leads to

$$
\|f\|_{L^{\infty}(\Omega)} \leq \sup _{x \in \Omega} \sqrt{\Phi(x, x)}\|f\|_{H_{t_{0}}} .
$$

Combining (32)-(34), we get

$$
\left\|K\left(I-P_{N}\right) f\right\|_{L^{2}(\Omega)} \leq M\left\|f-P_{N} f\right\|_{L^{\infty}(\Omega)} \leq M h_{X_{N}, \Omega}^{k}\|f\|_{H_{t_{0}}},
$$

where $M$ is a constant. Consequently, substituting the estimate (35) into (31) and letting $N \rightarrow \infty$, we complete the proof.

According to the classical results on the Tikhonov regularization for linear ill-posed problem (see, e.g., $[23,24])$ and in view of (1), it holds that

$$
\begin{aligned}
\left\|f-f_{\alpha, N, \epsilon}\right\| \leq & \left\|f-\left(K_{N}^{*} K_{N}+\alpha I\right)^{-1} K_{N}^{*} u\right\| \\
& +\left\|\left(K_{N}^{*} K_{N}+\alpha I\right)^{-1} K_{N}^{*}\left(u-u_{T}^{\epsilon}\right)\right\| \\
\leq & \left\|f-\left(K_{N}^{*} K_{N}+\alpha I\right)^{-1} K_{N}^{*} u\right\|+\frac{\epsilon}{2 \sqrt{\alpha}} .
\end{aligned}
$$

As in [35], we can estimate the noise-free term as follows:

$$
\begin{aligned}
\| f-( & \left.K_{N}^{*} K_{N}+\alpha I\right)^{-1} K_{N}^{*} u \| \\
= & \left\|f-\left(K_{N}^{*} K_{N}+\alpha I\right)^{-1} K_{N}^{*} K f\right\| \\
\leq & \left\|\left[I-\left(K_{N}^{*} K_{N}+\alpha I\right)^{-1} K_{N}^{*} K_{N}\right] f\right\| \\
& +\left\|\left(K_{N}^{*} K_{N}+\alpha I\right)^{-1} K_{N}^{*}\left(K_{N}-K\right) f\right\| \\
= & \left\|\left[I-\left(K_{N}^{*} K_{N}+\alpha I\right)^{-1} K_{N}^{*} K_{N}\right]\left(K^{*} K\right)^{\mu} w\right\| \\
& +\left\|\left(K_{N}^{*} K_{N}+\alpha I\right)^{-1} K_{N}^{*}\left(K_{N}-K\right) f\right\| \\
\leq & \|\left[I-\left(K_{N}^{*} K_{N}+\alpha I\right)^{-1} K_{N}^{*} K_{N}\right] \\
& \times\left[\left(K^{*} K\right)^{\mu}-\left(K_{N}^{*} K_{N}\right)^{\mu}\right] w \| \\
& +\left\|\left[I-\left(K_{N}^{*} K_{N}+\alpha I\right)^{-1} K_{N}^{*} K_{N}\right]\left(K_{N}^{*} K_{N}\right)^{\mu} w\right\| \\
& +\frac{1}{\sqrt{\alpha}}\left\|K\left(I-P_{N}\right) f\right\| \\
\leq & \left\|\left[\left(K^{*} K\right)^{\mu}-\left(K_{N}^{*} K_{N}\right)^{\mu}\right] w\right\| \\
+ & \alpha^{\mu} E+\frac{1}{\sqrt{\alpha}}\left\|K\left(I-P_{N}\right) f\right\| \\
&
\end{aligned}
$$

$$
\begin{aligned}
\leq & \left\|K^{*} K-K_{N}^{*} K_{N}\right\|^{\mu} E+\alpha^{\mu} E \\
& +\frac{1}{\sqrt{\alpha}}\left\|K\left(I-P_{N}\right) f\right\| \\
\leq & C\left\|K\left(I-P_{N}\right)\right\|^{2 \mu} E+\alpha^{\mu} E \\
& +\frac{1}{\sqrt{\alpha}}\left\|K\left(I-P_{N}\right) f\right\| \\
\leq & C\left\|K\left(I-P_{N}\right)\right\|^{2 \mu} E+\alpha^{\mu} E \\
& +\frac{1}{\sqrt{\alpha}}\left\|K\left(I-P_{N}\right)\right\|^{2 \mu+1},
\end{aligned}
$$

where $C$ is a constant. In view of the best possible error bound being $\epsilon^{2 \mu /(2 \mu+1)}$, the term $\left\|K\left(I-P_{N}\right)\right\|$ has to be chosen such that

$$
\left\|K\left(I-P_{N}\right)\right\| \leq \epsilon^{1 /(2 \mu+1)}
$$

From the above discussions, we have the following theorem.

Theorem 3. Under assumptions (5) and (38), there holds that

$$
\left\|f-f_{\alpha, N, \epsilon}\right\| \leq C \epsilon^{2 \mu /(2 \mu+1)}+\alpha^{\mu} E+\frac{\epsilon}{\sqrt{\alpha}} .
$$

Moreover, if the regularization parameter $\alpha$ is chosen by $\alpha=$ $O\left(\epsilon^{2 /(2 \mu+1)}\right)$, one then obtains the following estimate:

$$
\left\|f-f_{\alpha, N, \epsilon}\right\| \leq \widetilde{C} \epsilon^{2 \mu /(2 \mu+1)},
$$

where the constant $\widetilde{C}$ does not depend on $\epsilon$.

\section{Numerical Tests}

In this section, we present numerical results to illustrate the feasibility of the reconstruction method as described in the previous section.

In practical situation, we only can get the scattered noisy data of $u(\cdot, T)$, that is, $\left\{u_{\epsilon}\left(z_{1}, T\right), u_{\epsilon}\left(z_{2}, T\right), \ldots, u_{\epsilon}\left(z_{m}, T\right)\right\}$. As a result, instead of solving (25) we intend to deal with the following problem:

$$
\inf _{f \in V_{N}}\left\|K f-u_{\epsilon}(\cdot, T)\right\|_{\mathbb{R}^{m}}^{2}+\alpha\|f\|_{H_{t_{0}}(\Omega)}^{2},
$$

where

$$
\left\|K f-u_{\epsilon}(\cdot, T)\right\|_{\mathbb{R}^{m}}=\left\{\sum_{j=1}^{m}\left|K f\left(z_{j}\right)-u_{\epsilon}\left(z_{j}, T\right)\right|^{2}\right\}^{1 / 2} .
$$

Since $V_{N}=\operatorname{span}\left\{\Phi(\cdot, \xi) \mid \xi \in\left\{\xi_{1}, \xi_{2}, \ldots, \xi_{N}\right\}\right\}$, the minimizer $f_{\alpha, N, \epsilon}$ can be written as

$$
f_{\alpha, N, \epsilon}(\cdot)=\sum_{k=1}^{N} \tilde{\lambda}_{k} \Phi\left(\cdot, \xi_{k}\right)=\sum_{k=1}^{N} \tilde{\lambda}_{k} G\left(\cdot, t_{0} ; \xi_{k}\right), \quad \xi_{k} \in X_{N}
$$


From the definition of RKHS, it follows that

$$
\left\|f_{\alpha, N, \epsilon}\right\|_{H_{t_{0}}}^{2}=\sum_{j, k=1}^{N} \tilde{\lambda}_{k} \tilde{\lambda}_{j} G\left(\xi_{k}, t_{0} ; \xi_{j}\right) .
$$

In addition, we know that

$$
\begin{aligned}
& K G\left(z_{j}, t_{0} ; \xi_{k}\right) \\
& \quad=\int_{\Omega} G\left(z_{j}, T ; \xi\right) G\left(\xi, t_{0} ; \xi_{k}\right) d \xi=G\left(z_{j}, t_{0}+T ; \xi_{k}\right)
\end{aligned}
$$

for $j=1,2, \ldots, m$ and $k=1,2, \ldots, N$. Now, it is easy to see that the coefficient vector $\tilde{\lambda}=\left(\widetilde{\lambda}_{k}\right)_{k=1}^{N}$ satisfies the following linear system:

$$
\left(A^{*} A+\alpha B\right) \tilde{\lambda}=A^{*} u_{T}^{\epsilon}
$$

where $A$ is an $m \times N$ matrix, $A^{*}$ is the transpose of $A$, and $B$ is an $N \times N$ matrix defined by

$$
\begin{gathered}
A_{j, k}=G\left(z_{j}, t_{0}+T ; \xi_{k}\right), \quad j=1, \ldots, m, k=1, \ldots, N \\
B_{j, k}=G\left(\xi_{j}, t_{0} ; \xi_{k}\right), \quad j, k=1, \ldots, N .
\end{gathered}
$$

If we truncate Green's function to the former $J$ terms, matrix $B$ can be decomposed to the following product:

$$
B=L^{*} L,
$$

where $L^{*}$ denotes the conjugate transpose of $L$ and $L$ is given by

$$
\begin{gathered}
L=\left(L_{j, k}\right)=\left(\left[E_{\gamma}\left(-\lambda_{j}^{2} t_{0}^{\gamma}\right)\right]^{1 / 2} \varphi_{j}\left(\xi_{k}\right)\right), \\
j=1,2, \ldots, J, k=1,2, \ldots, N .
\end{gathered}
$$

Moreover, we turn to search for the minimizer $\tilde{\lambda}$ of the following functional:

$$
F_{\alpha}(\lambda):=\left\|A \lambda-u_{T}^{\epsilon}\right\|_{\mathbb{R}^{m}}^{2}+\|L \lambda\|_{\mathbb{R}^{I}}^{2}
$$

After obtaining the vector $\tilde{\lambda}$, we substitute it into (43) and then get the regularized approximation $f_{\alpha, N, \epsilon}$.

In our tests, the measurement points $\left\{z_{j}\right\}_{j=1}^{m}$, which are randomly generated by using the Matlab function $\operatorname{rand}(\cdot)$, are scattered in the domain $\Omega$. Now we generate the final measurement data at $T$ with noise by

$$
u_{T}^{\epsilon}=u(z, T)+\sqrt{\frac{2}{\pi}} \frac{\epsilon}{100} \times \operatorname{rand}(z) \times \operatorname{norm}(u(z, T)) \text {, }
$$

where $z=\left\{z_{j}\right\}_{j=1}^{m}$ are the measurement points and $\operatorname{rand}(z)$ generates a standard $m$-dimensional random vector. To evaluate the proposed method, we compute the relative error of the reconstructed solutions denoted by $R(f)$ :

$$
\begin{aligned}
& R(f)=\frac{\left\|f_{\alpha, N, \epsilon}(\cdot)-f(\cdot)\right\|_{l^{2}}}{\|f(\cdot)\|_{l^{2}}}, \quad \text { for 1-dimensional case, } \\
& R(f)=\frac{\left\|f_{\alpha, N, \epsilon}(\cdot)-f(\cdot)\right\|_{\infty}}{\|f(\cdot)\|_{\infty}}, \quad \text { for 2-dimensional case, }
\end{aligned}
$$

where $\|\cdot\|_{l^{2}}$ denotes the $l^{2}$ norm and $\|\cdot\|_{\infty}$ denotes the $\infty$ norm. Before we proceed, it is natural that we have to determine $t_{0}, X_{N}$, measurement points $\left\{z_{j}\right\}_{j=1}^{m}$, and observation time $T$ to define $A, B$. According to the convergence theorem, smaller $h_{X_{N}, \Omega}$ yields better numerical solution, which implies that we need to choose as large $N$ as possible. However, the ill posedness of the backward diffusion problem results in the ill condition of the matrix $A^{*} A$, which causes us not to implement the numerical computation of the inverse of $A^{*} A+\alpha B$ when the regularizing term $B$ is also ill conditioned. We use the following one-dimensional example to depict the change trend of the condition number of $B, \operatorname{cond}(B)$, with respect to $N$.

Example 1. Consider the following Dirichlet boundary value problem:

$$
\begin{gathered}
{ }_{0} D_{t}^{\gamma} u(x, t)=\frac{\partial^{2} u(x, t)}{\partial x^{2}}, \quad x \in(0,1), t \in[0, T], \\
u(x, 0)=x(1-x), \quad x \in[0,1] \\
u(0, t)=u(1, t)=0, \quad t \in[0,1] .
\end{gathered}
$$

The forward problem has a unique solution

$$
u(x, t)=\sum_{n=1}^{\infty} d_{n} E_{\gamma}\left(-n^{2} \pi^{2} t^{\gamma}\right) \sin n \pi x,
$$

where the coefficient

$$
\begin{aligned}
d_{n} & =2 \int_{0}^{1} u(x, 0) \sin n \pi x d x \\
& =\frac{4}{n^{3} \pi^{3}}\left[1-(-1)^{n}\right], \quad n=1,2, \ldots
\end{aligned}
$$

To clarify the numerical influence of some relative parameters but $\gamma$, we fix parameter $\gamma=1 / 2$ firstly.

In this test, we fix $m=59$ firstly. In Figure 1, for the cases of $t_{0} \in\{1 e-8,1 e-9,1 e-10\}$, we plot $\operatorname{cond}(B)$ versus the number of $N$ running from 10 to 100 , respectively. The displayed results in Figure 1 show that the condition number $\operatorname{cond}(B)$ increases exponentially as $N$ increases. Nevertheless, we can remove such influence of the ill condition of $B$ on numerical computation through modifying its small singular value as a fixed small constant $\widetilde{\epsilon}$. And in doing so, the numerical precision does not change significantly. Therefore, we can implement the proposed method without worrying so much about the size limitation of $N$. For the 


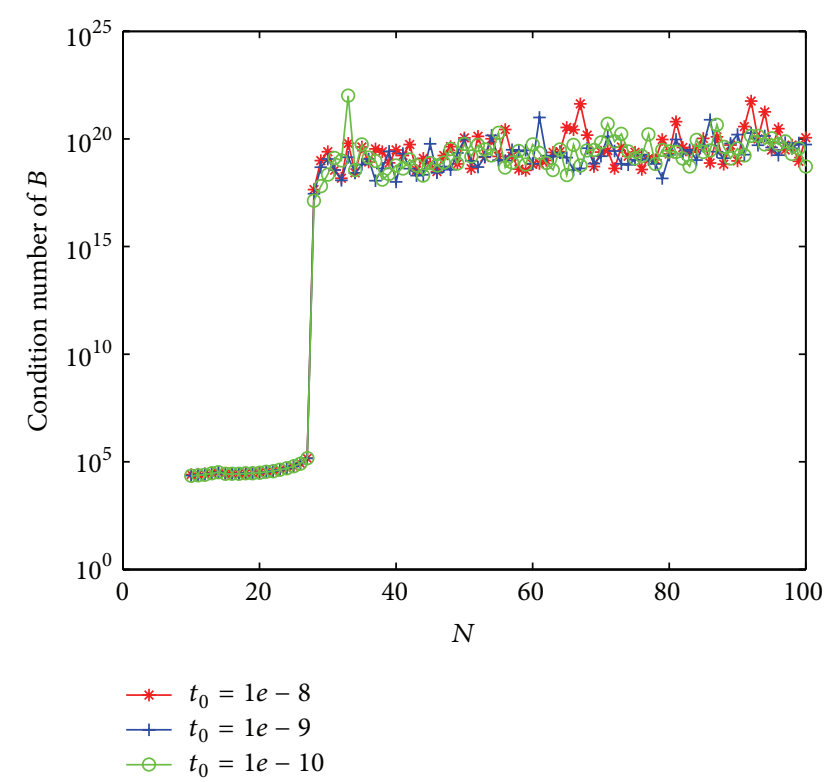

FIGURE 1: The effect of an increasing number of $N$ on the condition number of $B$ for the cases when $t_{0} \in\{1 e-8,1 e-9,1 e-10\}$.

TABle 1: Example 1: the relative errors with $t_{0}=1 e-10, N=59$, and $m=59$.

\begin{tabular}{lccc}
\hline$\epsilon$ & & $T$ & \\
& 1 & 5 & 10 \\
\hline $0.1 \%$ & 0.0143 & 0.0156 & 0.0177 \\
$1 \%$ & 0.0295 & 0.0239 & 0.0332 \\
$2 \%$ & 0.0388 & 0.0390 & 0.0387 \\
$5 \%$ & 0.0483 & 0.0441 & 0.0505 \\
$10 \%$ & 0.0528 & 0.0601 & 0.0703 \\
\hline
\end{tabular}

choice of $t_{0}$, we compare the computational results for some different $t_{0}$ 's and T's using exact final data $u(x, T)$ for Example 1. For this, a preset value for the regularization parameter $\alpha$ needs to be provided. Here, we simply choose $\alpha=10^{-5}\left(\max \left(A^{*} A\right) / \max (B)\right)$ as in [32], where $\max (C)=$ $\max _{i, j} c_{i j}$ for matrix $C=\left(c_{i j}\right)$. In the following computation, the Matlab code developed by Hansen $[36,37]$ is used to obtain the approximation solution for solving the discrete system (46). In addition, note that the Mittag-Leffler function is numerically realized by implementing the Matlab toolbox by Podlubny [38]. In Figure 2, we plot the relative errors for different $t_{0}, t_{0}=1 e-5,1 e-7,1 e-10$, versus the number of $N$ running from 10 to 100 for $\epsilon=0$ and $T=25$. The computational results show that, using smaller $t_{0}$, we have less relative error. In addition, we also see from Figure 2 that, with the increase of the number of $N$, the relative error becomes smaller firstly and then it remains steady after arriving to a certain extent.

We also need to consider the effect of the number $J$ of truncation term of Green's function and the number of measurement points $m$ on the numerical precision. With $T=$ 3 , we display the numerical results for several $m$ and $J$ as the

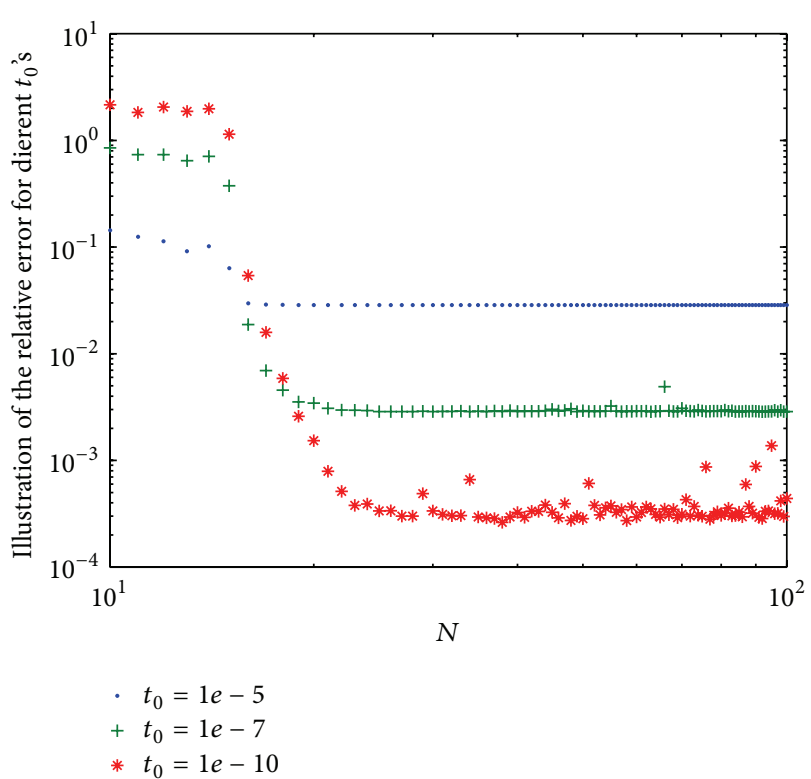

FIGURE 2: The effect of an increasing number of $N$ on the relative error for the cases when $t_{0} \in\{1 e-5,1 e-7,1 e-10\}$ with noise-free data.

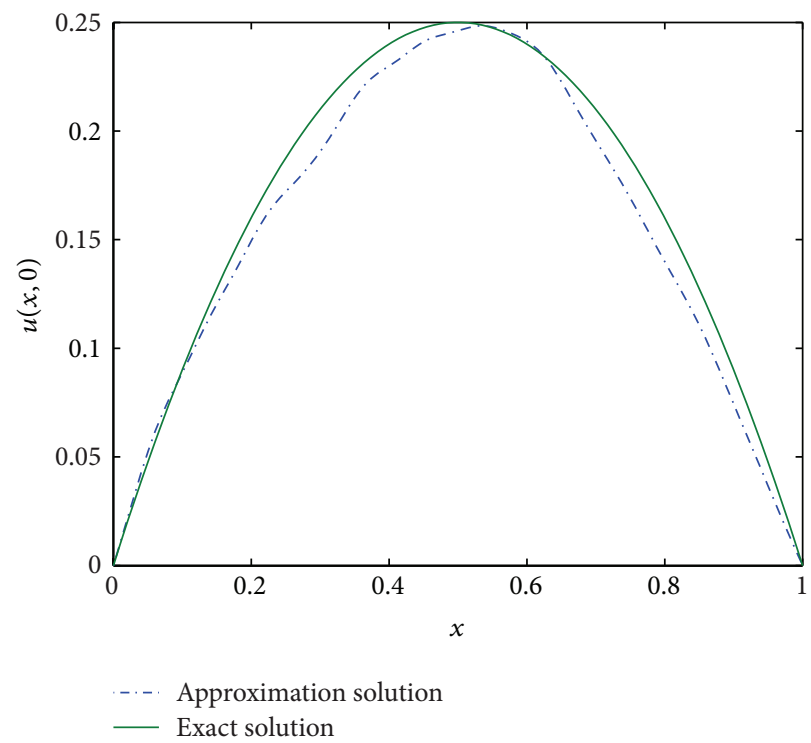

FIgURE 3: Exact solution $u(x, 0)$ and numerical solution for $\epsilon=20 \%$ with the final time $T=25$ and $m=59, N=59$, and $t_{0}=1 e-10$.

noise level $\epsilon=0$ in Table 2. It can be seen that when $J$ and $m$ become sufficiently large, the relative errors almost remain at the level $10^{-4}$.

Next, in the absence of the a priori information, we only evaluate the proposed algorithm in (46) for noisy data by using L-curve parameter choice method instead of that in Theorem 3. In Example 1, we fix $N=59, m=59$, and $t_{0}=1 e-10$. Table 1 reports the relative errors of $f_{\alpha, N, \epsilon}$ for different noise levels $\epsilon$ and final measurement times $T$. These numerical results for all noisy cases are satisfactory. In 
TABLE 2: Example 1: the relative errors with $t_{0}=1 e-10, N=59$, and $\epsilon=0 \%$.

\begin{tabular}{|c|c|c|c|c|c|c|}
\hline \multirow{2}{*}{$J$} & \multicolumn{6}{|c|}{$m$} \\
\hline & 19 & 29 & 39 & 49 & 59 & 69 \\
\hline 5 & $3.3721 e-3$ & $3.4356 e-3$ & $3.3769 e-3$ & $3.3737 e-3$ & $3.4008 e-3$ & $3.3724 e-3$ \\
\hline 10 & $1.0725 e-3$ & $1.5791 e-3$ & $9.9642 e-4$ & $9.9488 e-4$ & $9.9616 e-4$ & $9.9901 e-4$ \\
\hline 15 & $6.1811 e-4$ & $3.4322 e-4$ & $5.7376 e-4$ & $3.2938 e-4$ & $3.2952 e-4$ & $3.3259 e-4$ \\
\hline 20 & $5.0304 e-3$ & $3.5813 e-4$ & $8.9305 e-4$ & $4.7205 e-4$ & $9.8808 e-4$ & $5.4753 e-4$ \\
\hline 25 & $3.7310 e-3$ & $4.3286 e-4$ & $2.1742 e-4$ & $1.5605 e-4$ & $1.5743 e-4$ & $1.5375 e-4$ \\
\hline 30 & $5.9706 e-3$ & $2.6680 e-3$ & $1.6015 e-4$ & $1.5884 e-4$ & $2.0451 e-4$ & $1.3825 e-4$ \\
\hline
\end{tabular}

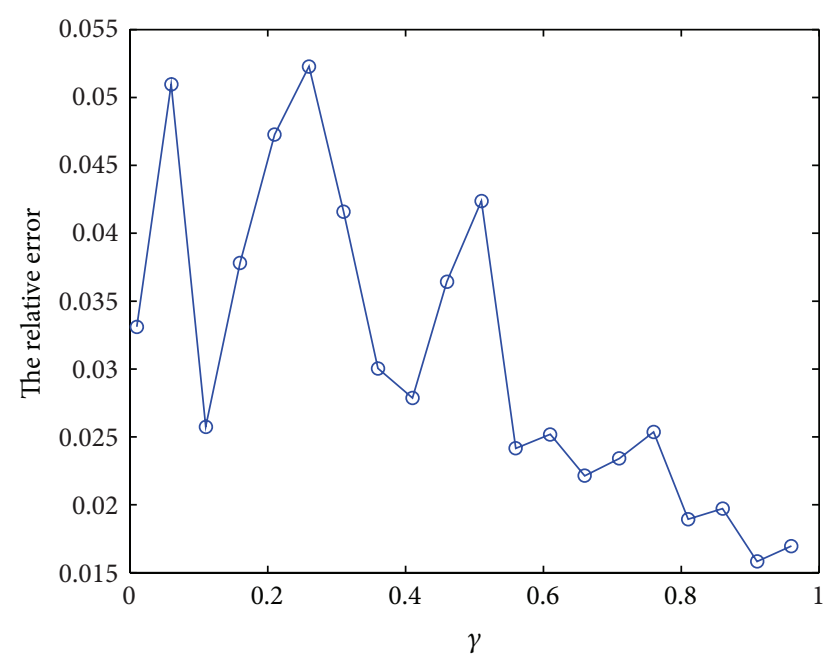

FIGURE 4: The relative error versus order $\gamma$ for Example 1 with $T=$ $0.5, m=59, N=59$, and $t_{0}=1 e-10$ for noise $\epsilon=1 \%$.

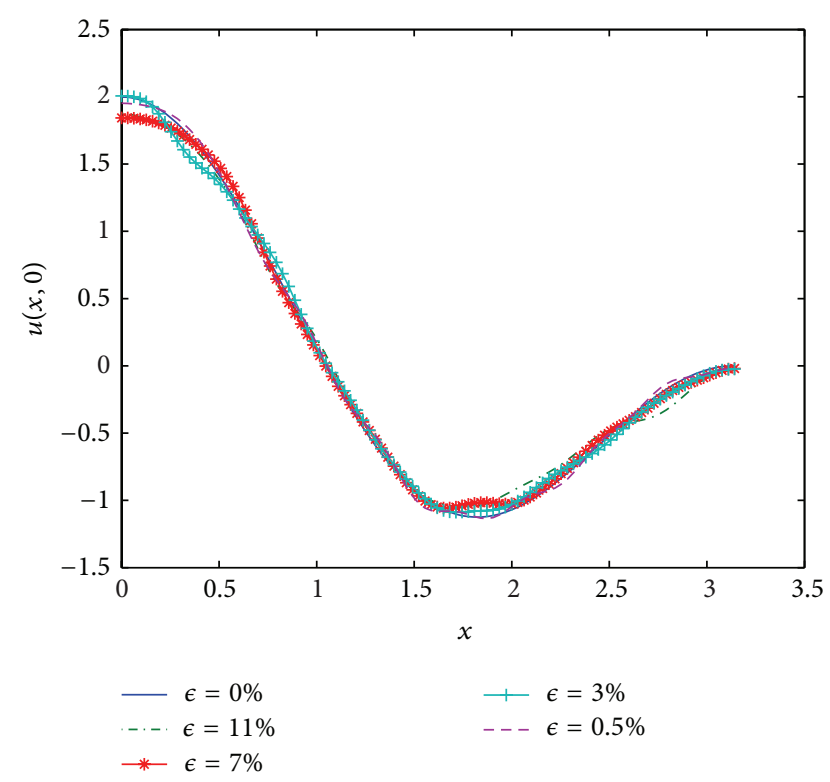

FIGURE 5: Exact solution $u(x, 0)$ and numerical solution for $\epsilon \epsilon$ $\{11 \%, 7 \%, 3 \%, 0.5 \%\}$ with the final time $T=0.1$ and $m=59$, $N=55$, and $t_{0}=1 e-10$.

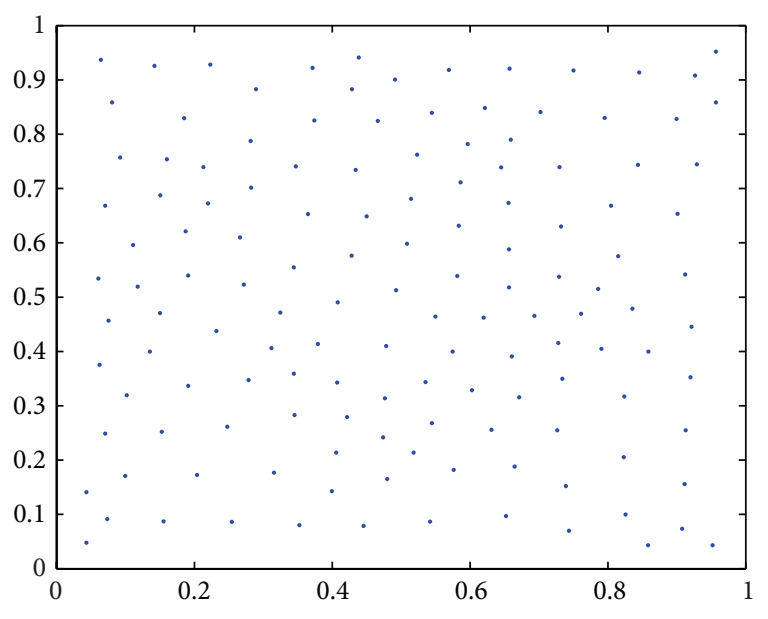

FIgURE 6: The illustration of the set $X_{N}$.

TABLE 3: Example 3: the relative errors with $t_{0}=1 e-10$ and $m=100$ for $\gamma=1 / 2$.

\begin{tabular}{lcccc}
\hline$\epsilon$ & \multicolumn{3}{c}{$T$} \\
& 1 & 2 & 5 & 10 \\
\hline $1 \%$ & 0.0131 & 0.0117 & 0.0138 & 0.0199 \\
$2 \%$ & 0.0118 & 0.0141 & 0.0229 & 0.0201 \\
$5 \%$ & 0.0272 & 0.0192 & 0.0304 & 0.0535 \\
\hline
\end{tabular}

general, it can been seen from Table 1 that, at the smaller $T$ and $\epsilon$, the numerical effects are better. In addition, when the measurement time $T=25$, the exact solution $u(x, 0)$ and the numerical solution $f_{\alpha, N, \epsilon}$ with the relative noise level $\epsilon=20 \%$ are displayed in Figure 3. It can be observed that the method works even for the case of $T=25$ with noise level $\epsilon=20 \%$ as well.

Finally, we hope to use Example 1 to show that the proposed algorithm is robust for order $\gamma$. For varying $\gamma$, we plot the relative error versus $\gamma$ in Figure 4 . The displayed results show that the numerical method is robust when $\gamma$ is varying. 

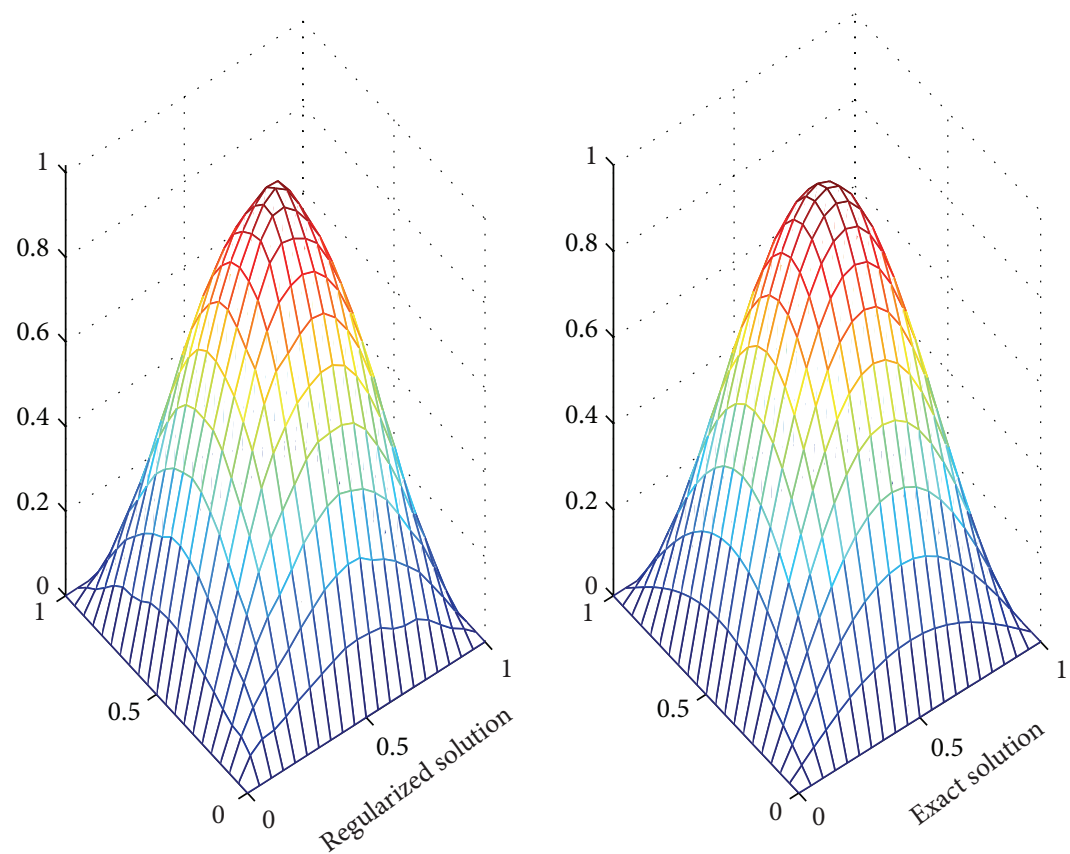

FIGURE 7: The comparison between the exact solution and regularized solution for Example 3.

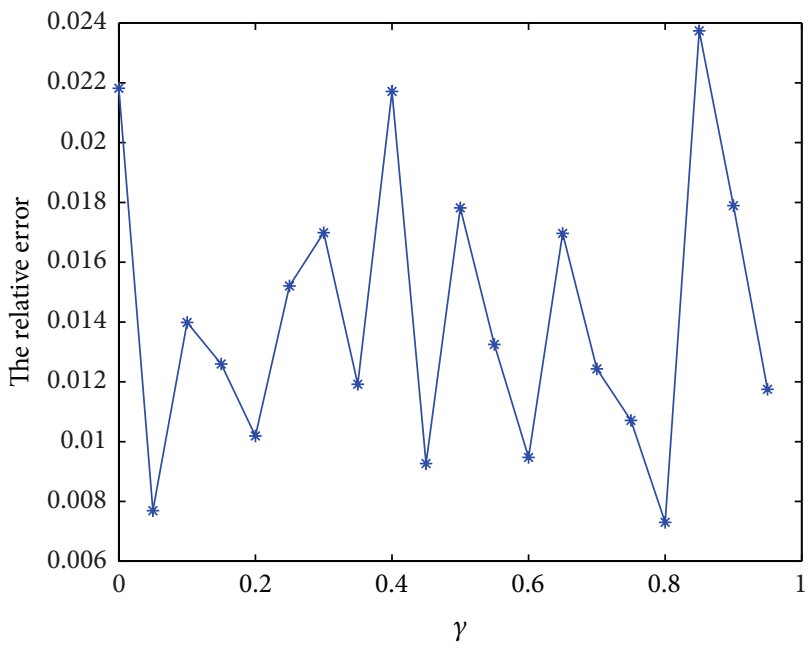

FIGURE 8: The relative error versus order $\gamma$ for Example 3 with $T=$ $1, m=100$, and $t_{0}=1 e-10$ for noise $\epsilon=1 \%$.

Example 2. Consider the following Neumann boundary value problem:

$$
\begin{gathered}
{ }_{0} D_{t}^{1 / 2} u(x, t)=\frac{\partial^{2} u(x, t)}{\partial x^{2}}, \quad x \in(0, \pi), t \in(0, T), \\
u(x, 0)=\cos x+\cos 2 x, \quad x \in[0, \pi], \\
u_{x}(0, t)=u_{x}(\pi, t)=0, \quad t \in[0, T] .
\end{gathered}
$$

The unique solution to (56) is given by

$$
u(x, t)=E_{1 / 2}\left(-t^{1 / 2}\right) \cos x+E_{1 / 2}\left(-4 t^{1 / 2}\right) \cos 2 x .
$$

In this example, we show the numerical results for $T=0.1$ under the setting $m=59, t_{0}=1 e-10$, and $N=55$. Figure 5 shows that the proposed method is capable of giving satisfactory results for the case of the Neumann boundary condition.

Example 3. We consider a two-dimensional fractional diffusion problem with the Dirichlet boundary value in $\Omega=$ $(0,1) \times(0,1)$ :

$$
\begin{gathered}
{ }_{0} D_{t}^{\gamma} u(x, y, t)=\Delta u, \quad x \in \Omega, t \in(0, T), \\
u(x, y, 0)=\sin \pi x \sin \pi y, \quad x \in \bar{\Omega}, \\
u(x, y, t)=0, \quad x \in \partial \Omega, t \in[0, T] .
\end{gathered}
$$

The initial distribution $u(x, y, 0)$ is to be recovered by using the exact solution

$$
u(x, y, t)=E_{\gamma}\left(-\pi^{2} t^{\gamma}\right) \sin \pi x \sin \pi y .
$$

We firstly display the choice of the set $X_{N}$ in Figure 6 . According to the analysis for 1-dimensional case about the number of $J$ and $m$, we only deal with the case of $t_{0}=1 e-$ 10 and $m=100$. The measurement points $\left\{x_{1}, x_{2}, \ldots, x_{m}\right\}$ are scattered in $\Omega$, which are generated using the Matlab command rand. We report the relative error of $f_{\alpha, N, \epsilon}$ for different final times $T$ and noise levels $\epsilon$ in Table 3 for $\gamma=$ $1 / 2$. The numerical comparison between exact solution and regularized solution is shown in Figure 7 . The numerical results show that the proposed method is acceptable for the 2-dimensional example. We also consider the influence of varying $\gamma$ on the numerical stability. The relative error versus $\gamma$ is plotted in Figure 8, from which we can see that the proposed method is robust about parameter $\gamma$. 


\section{Conclusion}

In this paper, in a reproducing kernel Hilbert space setting, we propose a numerical reconstruction method, namely, the discretized Tikhonov regularization method, to recover the initial temperature distribution of the backward fractional diffusion problem. The implementation of the proposed method is simple and easy. Numerical tests show that the method is efficient.

\section{Appendix}

\section{Reproducing the Kernel Hilbert Spaces and Positive Definite Kernels}

Most of the material in this appendix can be found in the excellent monograph [26]. For the readers' convenience we would like to repeat the theoretical results of RKHS. We are interested in linear vector spaces consisting of functions $f$ : $\Omega \rightarrow \mathbb{R}$ defined on a connected domain $\Omega$ of $\mathbb{R}^{d}$.

Definition A.1. Let $H$ be a Hilbert space consisting of functions $f: \Omega \rightarrow \mathbb{R} . H$ is called a reproducing kernel Hilbert space and a kernel $\Phi: \Omega \times \Omega \rightarrow \mathbb{R}$ is called a reproducing kernel for $H$ if

(i) $\Phi(\cdot, y) \in H$ for all $y \in \mathbb{R}^{d}$,

(ii) $f(y)=(f, \Phi(\cdot, y))_{H}$ for all $f \in H$ and all $y \in \mathbb{R}^{d}$,

where $(\cdot, \cdot)_{H}$ is the inner product of $H$.

The reproducing kernel of a RKHS is uniquely determined. According to [26], $H$ is a RKHS if and only if the point evaluation functionals are continuous, that is, $\delta_{y} \in H^{*}$ for all $y \in \Omega$. Also [26] discloses the connection between RKHS and positive definite kernels. Here, we call $\Phi$ symmetric if $\Phi(x, y)=\Phi(y, x)$ for all $x, y \in \Omega$.

Definition A.2. A continuous symmetric $\Phi: \Omega \times \Omega \rightarrow \mathbb{R}$ is called positive definite on $\Omega \subset \mathbb{R}^{d}$ if, for all $N \in \mathbb{N}$, all sets of pairwise distinct centers $X_{N}=\left\{\xi_{1}, \xi_{2}, \ldots, \xi_{N}\right\} \subset \Omega$; the quadratic form

$$
\begin{array}{r}
\sum_{j=1}^{N} \sum_{k=1}^{N} \lambda_{j} \lambda_{k} \Phi\left(\xi_{j}, \xi_{k}\right)>0, \\
\forall \lambda:=\left(\lambda_{1}, \lambda_{2}, \ldots, \lambda_{N}\right)^{T} \in \mathbb{R}^{N} \backslash\{0\} .
\end{array}
$$

If $\Phi$ is a symmetric positive definite kernel, then a unique RKHS in which the given kernel acts as the reproducing kernel can be constructed. Now, it follows from the definition of RKHS that

(i) $\Phi(x, y)=(\Phi(\cdot, x), \Phi(\cdot, y))_{H}$ for all $x, y \in \Omega$,

(ii) $\|f\|_{H}^{2}=\sum_{j=1}^{N} \sum_{k=1}^{N} \lambda_{j} \lambda_{k} \Phi\left(\xi_{j}, \xi_{k}\right)$ for all $f \in H$ in the form of $f=\sum_{k=1}^{N} \lambda_{k} \Phi\left(\cdot, \xi_{k}\right)$ with $\xi_{k} \in \Omega$.
For a symmetric positive definite kernel, introduce integral operator $T: L^{2}(\Omega) \rightarrow L^{2}(\Omega)$ by

$$
T v(x):=\int_{\Omega} \Phi(x, y) v(y) d y, \quad v \in L^{2}(\Omega), x \in \Omega .
$$

By [26, Proposition 10.28], $T$ maps $L^{2}(\Omega)$ continuously into the RKHS $H$ and is the adjoint of the embedding operator of the RKHS $H$ into $L^{2}(\Omega)$. For such an operator, Mercer's theorem [39] shows that $\Phi$ can be represented as

$$
\Phi(x, y)=\sum_{j=1}^{\infty} \rho_{j} \varphi_{j}(x) \varphi_{j}(y), \quad x, y \in \Omega,
$$

where $\left\{\rho_{j}\right\}$ are the nonnegative eigenvalues and $\varphi_{j}$ are the eigenfunctions of $T$. This allows us to derive the final characterization for RKHS $H$.

Theorem A.3. Suppose $\Phi$ is a symmetric positive definite kernel on a compact set $\Omega \subseteq \mathbb{R}^{d}$. Then the RKHS is given by

$$
H=\left\{f \in L^{2}(\Omega): \sum_{j=1}^{\infty} \frac{1}{\rho_{j}}\left|\left(f, \varphi_{j}\right)_{L^{2}(\Omega)}\right|^{2}<\infty\right\}
$$

and the inner product has the representation

$$
(f, g)_{H}=\sum_{n=1}^{\infty} \frac{1}{\rho_{j}}\left(f, \varphi_{j}\right)_{L^{2}(\Omega)}\left(g, \varphi_{j}\right)_{L^{2}(\Omega)}, \quad f, g \in H .
$$

For a finite set of points $X_{N}:=\left\{\xi_{1}, \xi_{2}, \ldots, \xi_{N}\right\} \subset \Omega$ and $f \in$ $\mathscr{H}$, consider the finite sum

$$
S_{f, X_{N}}(x)=\sum_{j=1}^{N} \lambda_{j} \Phi\left(x, \xi_{j}\right), \quad x \in \Omega,
$$

as an approximation of $f(x)$, which is actually the interpolant function of $f$. We also can consider $S_{f, X_{N}}$ in the following way: define a subspace $\mathscr{H}_{N}:=\operatorname{span}\left\{\Phi(\cdot, \xi) \mid \xi \in X_{N}\right\} \subset H$. We define the projection operator $P_{N}: \mathscr{H} \rightarrow \mathscr{H}_{N} \subset \mathscr{H}$ by

$$
P_{N}(f)(x)=S_{f, X_{N}}(x), \quad x \in \Omega,
$$

where $P_{N}$ is an orthogonal projection operator [26]. If the unknown function $f$ belongs to the related RKHS $H$, the error bound for the interpolant $S_{f, X_{N}}$ setup by the reproducing kernel $\Phi$ can be obtained by the following theorem.

Theorem A.4. Let domain $\Omega$ be open and bounded, satisfying an interior cone condition. Suppose that the $\Phi \in C^{2 k}(\Omega \times \Omega)$ is positive definite. If $f \in H$ and $h_{X_{N}, \Omega}$ is small enough, then

$$
\left|D^{\alpha} f(x)-D^{\alpha} S_{f, X_{N}}\right| \leq C h_{X_{N}, \Omega}^{k-|\alpha|}\|f\|_{H}, \quad x \in \Omega,
$$

where $C$ is a positive constant independent of $x$ and $f$ and $\alpha \epsilon$ $\mathbb{N}_{0}^{d}$ with $|\alpha| \leq k$. Here $D^{\alpha}$ denotes a derivative of order $\alpha=$ $\left(\alpha_{1}, \alpha_{2}, \ldots, \alpha_{d}\right)^{T}$; that is,

$$
D^{\alpha}:=\prod_{k=1}^{d} \frac{\partial^{\alpha_{k}}}{\partial x_{k}^{\alpha_{k}}}
$$




\section{Conflict of Interests}

The authors declare that there is no conflict of interests regarding the publication of this paper.

\section{Acknowledgments}

This work was supported by the Fundamental Research Funds for the Central Universities (ZYGX2011J104, SWJTU11BR078), the NSF of China (no. 11226040), and China Scholarship Council (no. 201208510083).

\section{References}

[1] K. Diethelm and N. J. Ford, "Numerical solution of the BagleyTorvik equation," BIT Numerical Mathematics, vol. 42, no. 3, pp. 490-507, 2002.

[2] F. Mainardi, "Some basic problems in continuum and statistical mechanics," in Fractals and Fractional Calculus in Continuum Mechanics, A. Carpinteri and F. Mainardi, Eds., pp. 291-348, Springer, Wien, Austria, 1997.

[3] I. Podlubny, Fractional Differential Equations, vol. 198, Academic Press, San Diego, Calif, USA, 1999.

[4] R. Gorenflo, F. Mainardi, D. Moretti, and P. Paradisi, "Time fractional diffusion: a discrete random walk approach," Nonlinear Dynamics, vol. 29, no. 1-4, pp. 129-143, 2002.

[5] C. Cattani and A. Ciancio, "Separable transition density in the hybrid model for tumor-immune system competition," Computational and Mathematical Methods in Medicine, vol. 2012, Article ID 610124, 6 pages, 2012.

[6] C. Cattani and G. Pierro, "On the fractal geometry of DNA by the binary image analysis," Bulletin of Mathematical Biology, vol. 75, no. 9, pp. 1544-1570, 2013.

[7] M. Li, S. C. Lim, C. Cattani, and M. Scalia, "Characteristic roots of a class of fractional oscillators," Advances in High Energy Physics, vol. 2013, Article ID 853925, 7 pages, 2013.

[8] M. Li, S. C. Lim, and S. Chen, "Exact solution of impulse response to a class of fractional oscillators and its stability," Mathematical Problems in Engineering, vol. 2011, Article ID 657839, 9 pages, 2011.

[9] M. Li, "Approximating ideal filters by systems of fractional order," Computational and Mathematical Methods in Medicine, vol. 2012, Article ID 365054, 6 pages, 2012.

[10] R. Gorenflo and F. Mainardi, Fractional Calculus: Integral and Differential Equations of Fractional Order: From, Fractals and Fractional Calculus, Carpinteri \& Mainardi, New York, NY, USA, 1997.

[11] Y. M. Lin and C. J. Xu, "Finite difference/spectral approximations for the time-fractional diffusion equation," Journal of Computational Physics, vol. 225, no. 2, pp. 1533-1552, 2007.

[12] F. Liu, V. V. Anh, I. Turner, and P. Zhuang, "Time fractional advection-dispersion equation," Journal of Applied Mathematics \& Computing, vol. 13, no. 1-2, pp. 233-245, 2003.

[13] W. R. Schneider and W. Wyss, "Fractional diffusion and wave equations," Journal of Mathematical Physics, vol. 30, no. 1, pp. 134-144, 1989.

[14] W. Wyss, "The fractional diffusion equation," Journal of Mathematical Physics, vol. 27, no. 11, pp. 2782-2785, 1986.

[15] V. V. Kulish and J. L. Lage, "Fractional-diffusion solution for transient local temperature and heat flux," Transactions of the ASME, vol. 122, pp. 372-376, 2000.
[16] K. B. Oldham and J. Spanier, The Fractional Calculus: Theory and Application of Differential and Integration to Arbitrary Order, Academic Press, 1974.

[17] K. B. Oldham and J. Spanier, "A general solution of the diffusion equation for semiinfinite geometries," Journal of Mathematical Analysis and Applications, vol. 39, pp. 655-669, 1972.

[18] K. B. Oldham and J. Spanier, "The replacement of Fick's law by a formulation involving semidifferentiation," Journal of Electroanalytical Chemistry, vol. 26, pp. 331-341, 1970.

[19] K. Sakamoto and M. Yamamoto, "Initial value/boundary value problems for fractional diffusion-wave equations and applications to some inverse problems," Journal of Mathematical Analysis and Applications, vol. 382, no. 1, pp. 426-447, 2011.

[20] J. J. Liu and M. Yamamoto, "A backward problem for the timefractional diffusion equation," Applicable Analysis, vol. 89, no. 11, pp. 1769-1788, 2010.

[21] C. X. Ren, X. Xu, and S. Lu, "Regularization by projection for a backward problem of the time-fractional diffusion equation," Journal of Inverse and III-Posed Problems, vol. 22, no. 1, pp. 121139, 2012.

[22] T. Takeuchi and M. Yamamoto, "Tikhonov regularization by a reproducing kernel Hilbert space for the Cauchy problem for an elliptic equation," SIAM Journal on Scientific Computing, vol. 31, no. 1, pp. 112-142, 2008.

[23] H. W. Engl, M. Hanke, and A. Neubauer, Regularization of Inverse Problems, vol. 375, Kluwer Academic Publishers Group, Dordrecht, The Netherlands, 1996.

[24] C. W. Groetsch, The Theory of Tikhonov Regularization for Fredholm Equations of the First Kind, vol. 105, Pitman, Boston, Mass, USA, 1984.

[25] I. Steinwart and A. Christmann, Support Vector Machines, Springer, New York, NY, USA, 2008.

[26] H. Wendland, Scattered Data Approximation, vol. 17 of Cambridge Monographs on Applied and Computational Mathematics, Cambridge University Press, Cambridge, UK, 2005.

[27] M. Pontil, "A note on different covering numbers in learning theory," Journal of Complexity, vol. 19, no. 5, pp. 665-671, 2003.

[28] J. Xian, S. P. Luo, and W. Lin, "Weighted sampling and signal reconstruction in spline subspaces," Signal Process, vol. 86, pp. 331-340, 2006.

[29] H. Hult, "Approximating some Volterra type stochastic integrals with applications to parameter estimation," Stochastic Processes and Their Applications, vol. 105, no. 1, pp. 1-32, 2003.

[30] I. Antoniou and K. Gustafson, "Wavelets and stochastic processes," Mathematics and Computers in Simulation, vol. 49, no. 1-2, pp. 81-104, 1999.

[31] N. Aronszajn, "Theory of reproducing kernels," Transactions of the American Mathematical Society, vol. 68, pp. 337-404, 1950.

[32] Y. C. Hon and T. Takeuchi, "Discretized Tikhonov regularization by reproducing kernel Hilbert space for backward heat conduction problem," Advances in Computational Mathematics, vol. 34, no. 2, pp. 167-183, 2011.

[33] W. Y. Wang, B. Han, and M. Yamamoto, "Inverse heat problem of determining time-dependent source parameter in reproducing kernel space," UTMS 2011-11, University of Tokyo, 2011.

[34] S. Saitoh, "Applications of Tikhonov regularization to inverse problems using reproducing kernels," Inverse Problems, vol. 73, Article ID 012019, 2007.

[35] P. Mathé and S. V. Pereverzev, "Discretization strategy for linear ill-posed problems in variable Hilbert scales," Inverse Problems, vol. 19, no. 6, pp. 1263-1277, 2003. 
[36] P. C. Hansen, Rank-Deficient and Discrete Ill-Posed Problems, SIAM, Philadelphia, Pa, USA, 1998.

[37] P. C. Hansen, "Regularization tools: a Matlab package for analysis and solution of discrete ill-posed problems," Numerical Algorithms, vol. 6, no. 1-2, pp. 1-35, 1994.

[38] I. Podlubny, "Mittag-Leffler function, The MATLAB routine," http://www.mathworks.com/matlabcentral/fileexchange.

[39] W. Pogorzelski, Integral Equations and Their Applications, vol. 1, Pergamon Press, Oxford, UK, 1966. 


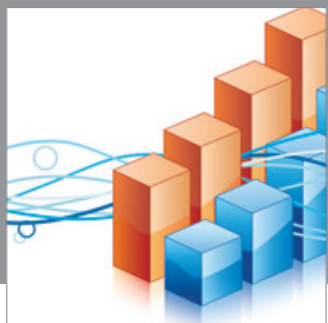

Advances in

Operations Research

mansans

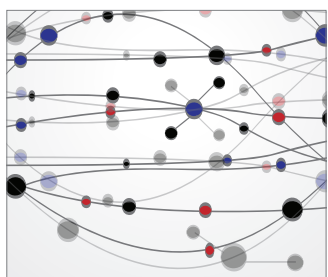

The Scientific World Journal
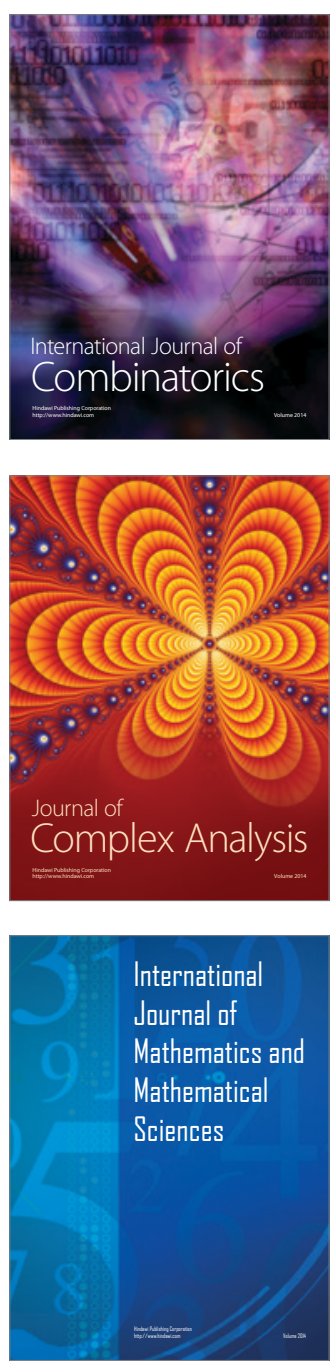
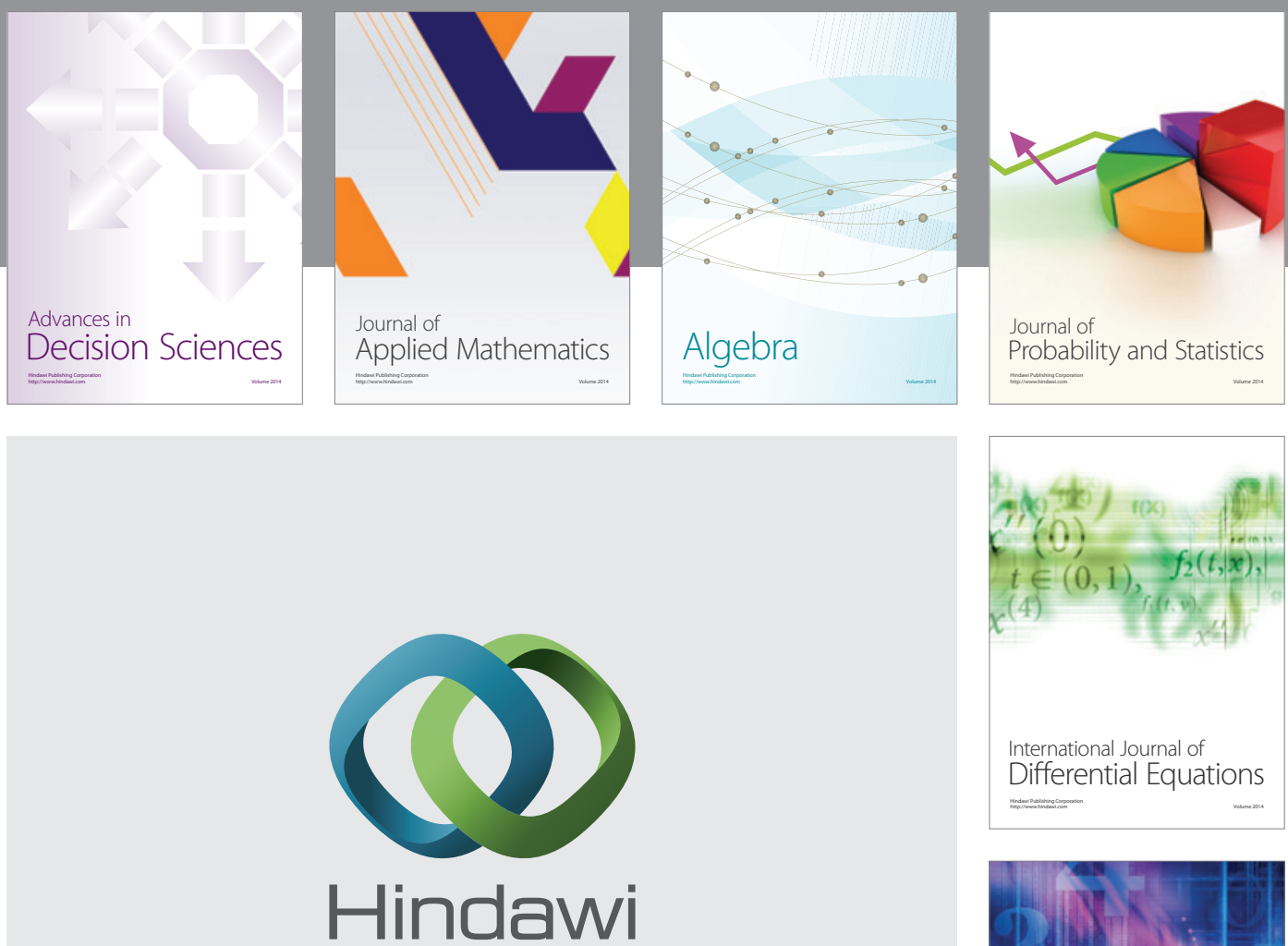

Submit your manuscripts at http://www.hindawi.com
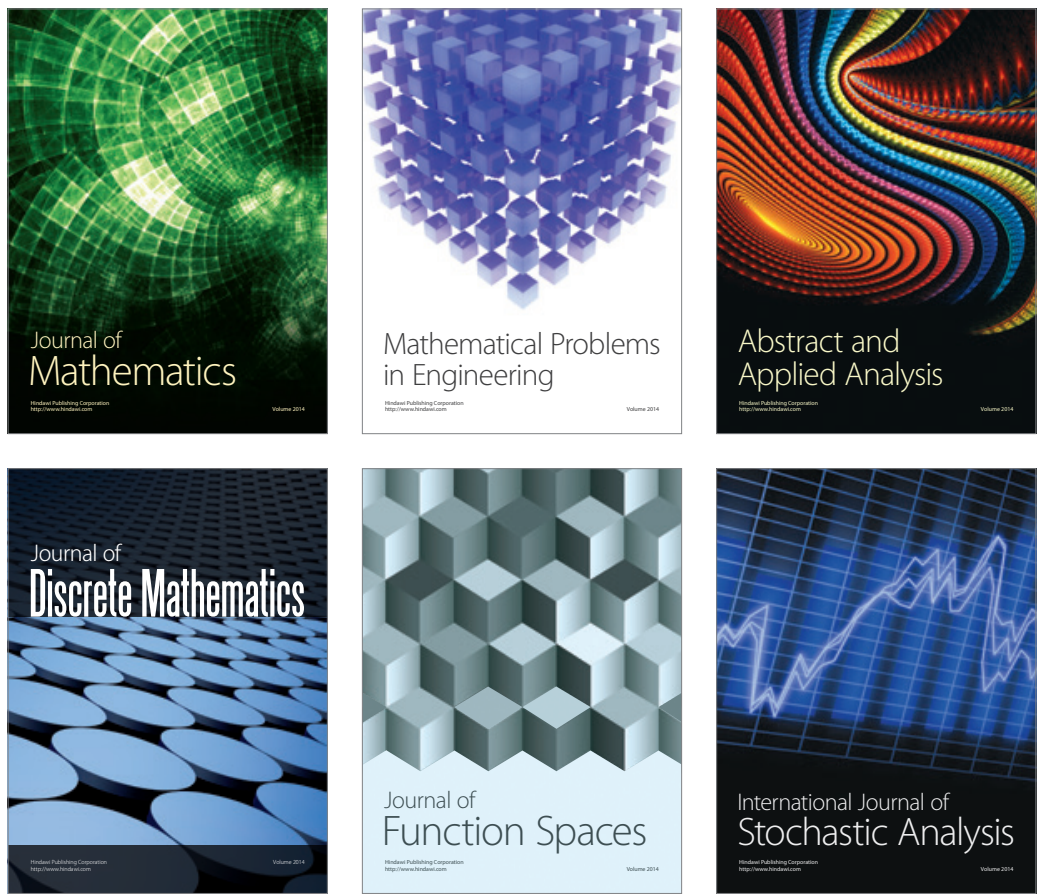

Journal of

Function Spaces

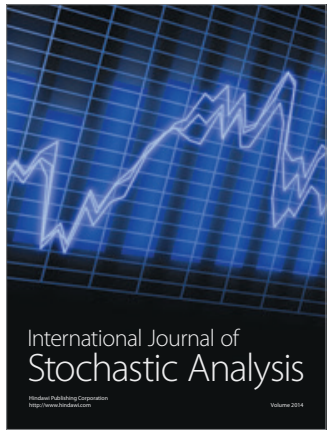

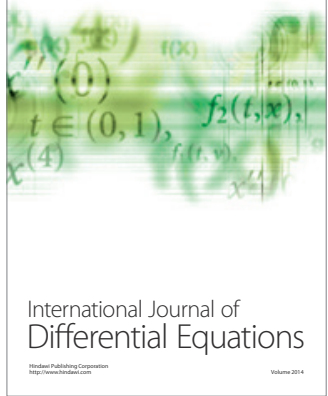
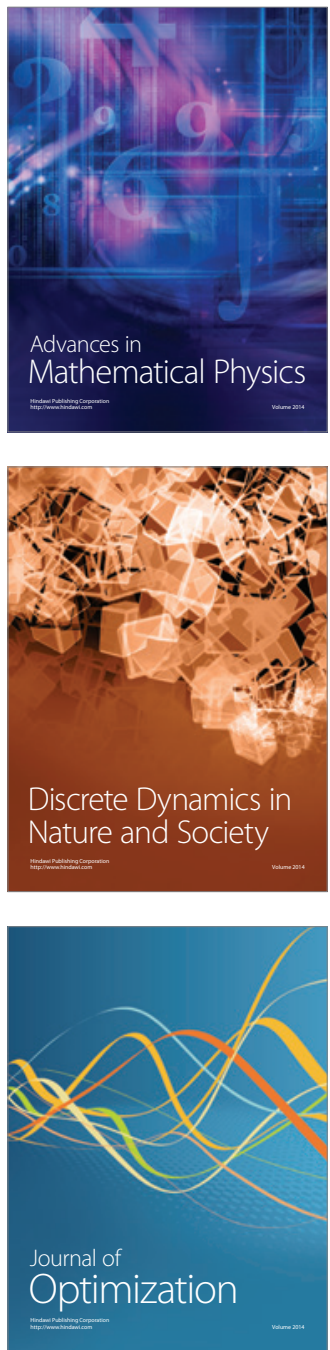